\title{
A class of new exact solutions of the system of PDE for the plane motion of viscous incompressible fluids in the presence of body force
}

\author{
Mushtaq Ahmed ${ }^{1}$ *, Waseem Ahmed Khan ${ }^{1}$ \\ ${ }^{1}$ Department of Mathematics, University of Karachi Karachi-75270, Pakistan \\ *Corresponding author E-mail: mushtaqku@yahoo.com
}

\begin{abstract}
The purpose of this paper is to indicate a class of exact solutions of the system of partial differential equations governing the steady, plane motion of incompressible fluid of variable viscosity with body force term to the right-hand side of Navier-Stokes equations. The class consists of the stream function $\psi$ characterized by the equation $\theta=f(r)+v(\psi)$ in polar coordinates $r$ and $\theta$ where $f$ and $v$ are continuously differentiable functions and the function $v(\psi)$ is such that $v^{\prime \prime}(\psi)=c v^{\prime 2}(\psi)$ where a non-zero constant is $c$ and overhead prime represents derivative with respect to $\psi$. When $c>0$ or $c<0$ we show exact solutions for given one component of the body force for both the cases when the function $f(r)$ is arbitrary and when it is not. For the arbitrary function case, $f(r)$ appears in the coefficient of a linear second order ordinary differential equation showing a large numbers of solutions of this equation. This in turn establishes an infinite set of exact solutions to the problem concerned however; we show three examples of such exact solutions. The alternate case fixes $f(r)$ and provides viscosity as derivative of temperature function for $c>0$ and $c<0$. Anyhow, we find an infinite set of streamlines, the velocity components, viscosity function, generalized energy function and temperature distribution.
\end{abstract}

Keywords: Some Exact Solutions in the Presence of Body Force; Exact Solutions to the Flow Equations of Incompressible Fluids; Exact Solutions of Variable Viscosity Fluids; Navier-Stokes Equations with Body Force.

\section{Introduction}

The basic system of partial differential equations (PDE) for the motion of a viscous fluid consists of the equation of continuity, Navier-Stokes equations (NSE) and energy equation. As the Navier-Stokes equations have base on Newton's law, therefore it allows us to add body forces term to right-hand side of it in addition to surface force. The examples of the body forces are constant gravity force, coriolis force, centrifugal force etc. In the presence of body force the basic dimensionless form of system of PDE's for the steady motion of incompressible fluid of variable viscosity in tensor notation are

Continuity

$$
\frac{\partial v_{i}}{\partial x_{i}}=0
$$

NSE

$$
\left(v_{j} \frac{\partial v_{i}}{\partial x_{j}}\right)=F_{i}-\frac{\partial p}{\partial x_{i}}+\frac{1}{R_{e}} \frac{\partial}{\partial x_{j}}\left\{\mu\left(\frac{\partial v_{i}}{\partial x_{j}}+\frac{\partial v_{j}}{\partial x_{i}}\right)\right\}
$$

Energy

$$
\left(v_{j} \frac{\partial T}{\partial x_{j}}\right)=\frac{1}{R_{e} P_{r}} \frac{\partial}{\partial x_{i}}\left(\frac{\partial T}{\partial x_{i}}\right)+\frac{\mu E_{c}}{R_{e}} \frac{\partial v_{i}}{\partial x_{j}}\left(\frac{\partial v_{i}}{\partial x_{j}}+\frac{\partial v_{j}}{\partial x_{i}}\right)
$$

where $\mathbf{F}=\left(F_{1}\left(x_{i}\right), F_{2}\left(x_{i}\right), F_{3}\left(x_{i}\right)\right)$ is the body force per unit mass, $\mathbf{v}=\left(v_{1}\left(x_{i}\right), v_{2}\left(x_{i}\right), v_{3}\left(x_{i}\right)\right)$ the fluid velocity, $p=p\left(x_{i}\right)$ is pressure, $\rho=\rho\left(x_{i}\right)$ the fluid density, the coefficients of viscosity $\mu>0$, the space coordinates $x_{i}$ and $i, j \in\{1,2,3\}$. The dimensionless quantities $R_{e}, P_{r}$ and $E_{c}$ are the Reynolds number, the Prandtl number and the Eckert number respectively. The non-dimensional parameters used in equations (2-3) with constant thermal conductivity $k$ are mentioned in [1-2].

For the plane Cartesian space case we take $i, j \in\{1,2\}, x_{1}=x$, $x_{2}=y, v_{1}=u(x, y), v_{2}=v(x, y), F_{1}=F_{1}(x, y), F_{2}=F_{2}(x, y)$ in equations (1-3) and obtain

$u_{x}+v_{y}=0$

$u u_{x}+v u_{y}=F_{1}-p_{x}+\frac{1}{\mathrm{R}_{e}}\left[\left(2 \mu u_{x}\right)_{x}+\left\{\mu\left(u_{y}+v_{x}\right)\right\}_{y}\right]$

$u v_{x}+v v_{y}=F_{2}-p_{y}+\frac{1}{\mathrm{R}_{\mathrm{e}}}\left[\left(2 \mu v_{y}\right)_{y}+\left\{\mu\left(u_{y}+v_{x}\right)\right\}_{x}\right]$

$u T_{x}+v T_{y}=\frac{1}{\mathrm{R}_{\mathrm{e}} \mathrm{P}_{\mathrm{r}}}\left(T_{x x}+T_{y y}\right)$

$+\frac{\mathrm{E}_{\mathrm{c}}}{\mathrm{R}_{\mathrm{e}}}\left[2 \mu\left(u_{x}{ }^{2}+v_{y}{ }^{2}\right)+\mu\left(u_{y}+v_{x}\right)^{2}\right]$ 
The solution of the equation (4) demands the existence of a stream function $\psi(x, y)$ such that

$u=\frac{\partial \psi}{\partial y} \quad v=-\frac{\partial \psi}{\partial x}$

However the nonlinear terms in equations (5-7) offers a great difficulty for exact solutions. In order to handle nonlinearities some coordinates transformation techniques and dimension analysis methods have proved helpful for some exact solutions of NSE with surface force. The readers interested in these methods/techniques may refer to [3]-[16] and the references therein. For a class of some exact solutions of NSE with body force we refer [2] and the references therein.

The objective of this communication is to obtain a class of exact solutions of the problem of the steady plane motion of incompressible fluid of variable viscosity in the presence of body force with a new characterization of streamlines. To achieve the aim we transform the fundamental flows equations from Cartesian space $(x, y)$ into a curvilinear coordinates $(\phi, \psi)$ with Martin's definition. Martin [17] defined the curvilinear coordinate lines $\psi=$ const. as streamlines and left the curvilinear coordinate lines $\phi=$ const. arbitrary. We will refer it as Martin's system $-(\phi, \psi)$. As the coordinate $\phi$ is arbitrary in Martin's system, therefore, we take $\phi=r(x, y)$ to achieve our plan and we characterize the streamlines of the class of flows under consideration by

$\theta-f(r)=$ const

Where $f(r)$ is a continuously differentiable function, $r, \theta$ the polar coordinates. As $\psi=$ const. are the streamlines therefore, for the class of flows under consideration of this communication we take

$$
\theta=f(r)+v(\psi)
$$

With $v$ as a continuously differentiable function of $\psi$ such that $v^{\prime \prime}(\psi)=c v^{\prime 2}(\psi)$ where a non-zero constant is $c$ and overhead prime represents derivative with respect to $\psi$.

We organize this paper as follow: In section (2), we transform the fundamental non-dimensional flow equations from Cartesian space $(x, y)$ into Martin's system $(\phi, \psi)$. In section (3), we find exact solution taking $\phi=r(x, y)$. In last section, we present conclusion.

\section{Fundamental flow equations in Martin's system}

Following [1-3], we reduce the basic system Eqs. (5-7) to a convenient form by introducing the vorticity function $w$ and the total energy function $L$ defined by

$$
\begin{aligned}
w & =v_{x}-u_{y} \\
L & =p+\frac{1}{2}\left(u^{2}+v^{2}\right)-\frac{2 \mu u_{x}}{R_{e}}
\end{aligned}
$$

And find

$$
\begin{aligned}
& -v w=F_{1}-L_{x}+\frac{1}{\mathrm{R}_{\mathrm{e}}} A_{y} \\
& u w=F_{2}-L_{y}-\frac{1}{R_{e}} B_{y}+\frac{1}{\mathrm{R}_{\mathrm{e}}} A_{x}
\end{aligned}
$$

$$
u T_{x}+v T_{y}=\frac{1}{\mathrm{R}_{\mathrm{e}} \mathrm{P}_{\mathrm{r}}}\left(T_{x x}+T_{y y}\right)+\frac{\mathrm{E}_{\mathrm{c}}}{\mathrm{R}_{\mathrm{e}}} \frac{1}{4 \mu}\left(B^{2}+4 A^{2}\right)
$$

Where

$$
A=\mu\left(u_{y}+v_{x}\right) \quad B=4 \mu u_{x}
$$

Now we transform Eqs. (13-15) into Martin's system $(\phi, \psi)$ through transformation

$$
x=x(\phi, \psi), y=y(\phi, \psi)
$$

Such that the Jacobian $J=\frac{\partial(x, y)}{\partial(\phi, \psi)} \neq 0$ of the transformation is finite. At a common point $P(x, y)$ let $\alpha$ be the angle between the tangents to the streamlines lines $\psi=$ const. and the curves $\phi=$ const. then we have shown in [1-2] that Eqs. (13-15) in Martin's system are following

$$
\begin{aligned}
& -R_{e} w J E=R_{e} J \sqrt{E}\left[-F\left(F_{1} \cos \alpha+F_{2} \sin \alpha\right)+J\left(F_{1} \sin \alpha-F_{2} \cos \alpha\right)\right] \\
& +R_{e} J E L_{\psi}+A_{\phi}\left(\left(F^{2}-J^{2}\right) \cos 2 \alpha-2 F J \sin 2 \alpha\right) \\
& \left.+E A_{\psi}(J \sin 2 \alpha-F \cos 2 \alpha)\right)-B_{\phi}\left(\frac{1}{2}\left(F^{2}-J^{2}\right) \sin 2 \alpha+F J \cos 2 \alpha\right)
\end{aligned}
$$$$
+E B_{\psi}\left(\frac{1}{2} F \sin 2 \alpha+J \cos ^{2} \alpha\right),
$$

$0=R_{e} J \sqrt{E}\left[F_{1} \cos \alpha+F_{2} \sin \alpha\right]-R_{e} J L_{\phi}+E A_{\psi} \cos 2 \alpha$

$-A_{\phi}[F \cos 2 \alpha-J \sin 2 \alpha]+$

$B_{\phi}\left(\frac{1}{2} F \sin 2 \alpha-J \sin ^{2} \alpha\right)-\frac{E B_{\psi}}{2} \sin 2 \alpha$,

$\frac{1}{\mathrm{JR}_{\mathrm{e}} \mathrm{P}_{\mathrm{r}}}\left[\left(\frac{G T_{\phi}-F T_{\psi}}{J}\right)_{\phi}+\left(\frac{E T_{\psi}-F T_{\phi}}{J}\right)_{\psi}\right]$

$=-\frac{\mathrm{E}_{\mathrm{c}}}{\mathrm{R}_{\mathrm{e}}} \frac{1}{4 \mu}\left(B^{2}+4 A^{2}\right)+\frac{T_{\phi}}{J}$

Where the coefficients of first fundamental form are

$$
E=x_{\phi}^{2}+y_{\phi}^{2}, F=x_{\phi} x_{\psi}+y_{\phi} y_{\psi}, G=\left(x_{\psi}\right)^{2}+\left(y_{\psi}\right)^{2}
$$

And

$$
J= \pm \sqrt{E G-F^{2}}
$$

$B(\phi, \psi)=\frac{4 \mu}{E J^{3}}\left[E_{\phi}(F \sin \alpha+J \cos \alpha)^{2}-2 E(F \sin \alpha+J \cos \alpha)\right.$

$\left.\left(F_{\phi} \sin \alpha+J_{\phi} \cos \alpha\right)+E^{2}\left(J_{\psi /} \sin 2 \alpha+G_{\phi} \sin ^{2} \alpha\right)\right]$,

$A(\phi, \psi)=\mu\left[-\frac{(F \cos \alpha-J \sin \alpha)}{4 E^{2} J^{5}}\left\{E_{\phi}\left(2 E J^{3} \cos \alpha+F \sqrt{E} \sin \alpha\right)\right.\right.$

$\left.-4 E^{2} J^{2} J_{\phi} \cos \alpha-2 E \sqrt{E} F_{\phi} \sin \alpha+E \sqrt{E} E_{\psi} \sin \alpha\right\}$

$+\frac{\cos \alpha}{2 J^{3}}\left\{E_{\psi}(F \sin \alpha+J \cos \alpha)-2 E J_{\psi} \cos \alpha-E G_{\phi} \sin \alpha\right\}$ 


$$
\begin{aligned}
& +\frac{(F \sin \alpha+J \cos \alpha)}{2 E J^{3}}\left\{\left(J E_{\phi}-2 E J_{\phi}\right) \sin \alpha\right. \\
& \left.+\cos \alpha\left[-F E_{\phi}+2 E F_{\phi}-E E_{\psi}\right]\right\} \\
& -\frac{\sin \alpha}{2 J^{3}}\left\{\left(E_{\psi}(J \sin \alpha-F \cos \alpha)-2 E J_{\psi} \sin \alpha+E G_{\phi} \cos \alpha\right\}\right] \\
& w=\frac{(F \sin \alpha+J \cos \alpha)}{2 E J^{3}}\left\{\left(J E_{\phi}-2 E J_{\phi}\right) \sin \alpha\right. \\
& \left.+\cos \alpha\left[-F E_{\phi}+2 E F_{\phi}-E E_{\psi}\right]\right\} \\
& \left.-\frac{\sin \alpha}{2 J^{3}}\left\{E_{\psi}(J \sin \alpha-F \cos \alpha)-2 E J_{\psi} \sin \alpha+E G_{\phi} \cos \alpha\right\}\right] \\
& +\frac{(F \cos \alpha-J \sin \alpha)}{4 E^{2} J^{5}}\left\{E_{\phi}\left(2 E J^{3} \cos \alpha+F \sqrt{E} \sin \alpha\right)\right. \\
& -\left[\frac{\cos \alpha}{2 J^{3}}\left\{E_{\psi}(F \sin \alpha+J \cos \alpha)-2 E J_{\psi} \cos \alpha-E G_{\phi} \sin \alpha\right\}\right] \\
& \left.4 E^{2} J^{2} J_{\phi} \cos \alpha-2 E \sqrt{E} F_{\phi} \sin \alpha+E \sqrt{E} E_{\psi} \sin \alpha\right\}
\end{aligned}
$$

Thus, the basic transformed equations into Martin's system are Eqs. (18-25).

\section{Exact solutions}

As the coordinate $\phi$ is arbitrary in Martin's system $(\phi, \psi)$, therefore, we take

$$
\phi=r(x, y)
$$

Where

$$
x=r \operatorname{Cos} \theta, y=r \operatorname{Sin} \theta
$$

Utilizing equations (26-27) and writing the fundamental equations equation (18-25) in terms of independent variables $r$ and $\psi$, we get

$$
\begin{aligned}
& q=\frac{\sqrt{E}}{J} \\
& -R_{e} w=-R_{e} J F_{2}+R_{e} L_{\psi}-J A_{r}+\sqrt{E-1} A_{\psi}+B_{\psi} \\
& 0=R_{e}\left(F_{1}+F_{2} \sqrt{E-1}\right)-R_{e} L_{r}+\frac{A_{\psi}(2-E)}{J} \\
& +A_{r} \sqrt{E-1}-\frac{\sqrt{E-1} B_{\psi}}{J} \\
& J T_{r r}-2 \sqrt{E-1} T_{v r} v^{\prime}+\frac{E}{J} T_{v v}\left(v^{\prime}\right)^{2}+\left(J_{r}-\frac{E_{\psi}}{2 \sqrt{E-1}}-R_{e} P_{r}\right) T_{r} \\
& +\left(\frac{E_{\psi}}{J}-\frac{E E_{r}}{2 \sqrt{E-1}}-\frac{E J_{\psi}}{J^{2}}+\frac{E}{J}\left(\frac{v^{\prime \prime}}{v^{\prime}}\right)\right) T_{v} v^{\prime}=-\frac{J E_{c} P_{r}}{4 \mu}\left(B^{2}+4 A^{2}\right) \\
& w=\left[\frac{f^{\prime}(r)}{r}+f^{\prime \prime}(r)\right]\left(\frac{1}{v^{\prime}(\psi)}\right)+\left[\frac{1}{r^{2}}+\left\{f^{\prime}(r)\right\}^{2}\right]\left(\frac{v^{\prime \prime}(\psi)}{\left\{v^{\prime}(\psi)\right\}^{3}}\right)
\end{aligned}
$$

Where

$$
\begin{aligned}
& A(r, \psi)=\frac{\mu}{J}\left[\frac{-2 J_{r} \sqrt{E-1}}{J}+\frac{E_{r}}{2 \sqrt{E-1}}+\frac{-(2-E) J_{\psi}}{J^{2}}\right] \\
& B(r, \psi)=4 \mu \frac{1}{J^{3}}\left[-J J_{r}+\sqrt{E-1} J_{\psi}\right] \\
& E=1+r^{2}\left[f^{\prime}(r)\right]^{2} \\
& F=J \sqrt{E-1} \\
& G=r^{2} v^{\prime}(\psi)^{2} \\
& J=r v^{\prime}(\psi) \\
& \cos \alpha=\frac{1}{\sqrt{E}}
\end{aligned}
$$

We are using $q$ is the magnitude of velocity vector $\mathbf{q}=(u, v)$ for the plane motion.

In order to determine the solution of the flow equations (28-39), we follow [1-3] and construct the following equation using the natural integrability condition $L_{r \mu}=L_{\psi r}$ on equations (29) and (30).

$r v^{\prime} A_{r r}-2 r f^{\prime} A_{r \psi}-\frac{\left[1-r^{2}\left(f^{\prime}\right)^{2}\right]}{r v^{\prime}} A_{\psi \psi}+v^{\prime} A_{r}-A_{\psi}\left(f^{\prime}+r f^{\prime \prime}\right)$

$-\left\{B_{r}-\frac{f^{\prime} B_{\psi}}{v^{\prime}}\right\}_{\psi}=R_{e} w_{r}+R_{e}\left(F_{1}+F_{2} r\left(f^{\prime}\right)\right)_{\psi}-R_{e}\left(r v^{\prime} F_{2}\right)_{r}$

This equation involves the functions $f(r), v(\psi)$, the body force components $F_{1}(r, \psi), F_{2}(r, \psi)$ and the viscosity $\mu$. Once a solution of this equation (40) is determined, the function $L$ and temperature distribution $T$ are determined from equations (29-30) and (31), respectively, the pressure from equation (12) and velocity components from (8).

In order to attempt for solution of equation (40) the first inspiration that we take is from [2] which guides to focus on the vorticity function $w$ and write it as a product function of independent variables $r$ and $\psi$ through

$v^{\prime \prime}=c v^{\prime 2}$

Where $c$ is constant. The case for $c=0$ has already discussed in [2]. The case when $c>0$ or $c<0$ the solution of equation (41) is

$$
v=\frac{1}{c} \ln \left[\frac{-1}{c\left(k_{1} \psi+k_{2}\right)}\right]
$$

Where $k_{1}$ and $k_{2}$ are constant of integration. Utilizing equation (42) in equations (32-34), we have

$$
\begin{aligned}
& w=\left(\frac{1}{v^{\prime}}\right)\left[\frac{M^{\prime}}{r}+\frac{c\left(1+M^{2}\right)}{r^{2}}\right], \\
& A=\frac{\mu}{r^{2} v^{\prime}}\left[r M^{\prime}-2 M-c\left(1-M^{2}\right)\right]
\end{aligned}
$$

And

$B=\frac{4 \mu}{r^{2} v^{\prime}}[-1+c M]$

Where 
$M(r)=r f^{\prime}(r)$

Other observation about the compatibility equation (40) is that it involves functions $A, B$ which depends upon the viscosity function $\mu$, the function $f(r)$ and derivative of $f(r)$ therefore it is extremely difficult to solve it analytically. The second guidance that we take from [2] is that the equation resulting from compatibility condition provide solution on eliminating $\mu$ from the functions $A$ and $B$. Therefore we eliminate $\mu$ from equation (44) and (45) by introducing function $Y(r)$ through

$$
A=Y(r) \quad B
$$

Where

$$
Y(r)=\frac{r M^{\prime}-2 M-c\left(1-M^{2}\right)}{4(-1+c M)} \neq 0
$$

Inserting equation (47) in equation (40), we have

$r Y B_{r r}-$

$(1+2 M Y) B_{v r}+B_{v v}\left(-\frac{\left(1-M^{2}\right) Y}{r}+\frac{M}{r}\right)+B_{v}\left[-2 M Y^{\prime}-Y M^{\prime}\right]$

$+B_{r}\left(2 r Y^{\prime}+Y\right)+B\left(r Y^{\prime \prime}+Y^{\prime}\right)$

$=R_{e}\left(\frac{1}{v^{\prime 2}}\right)\left[\frac{M^{\prime}}{r}+\frac{c\left(1+M^{2}\right)}{r^{2}}\right]^{\prime}+R_{e}\left(F_{1}+M F_{2}\right)_{v}-R_{e}\left(r F_{2}\right)$,

Since the solution of equation (49) is to lead us for the function $L$ from equations (29-30) and temperature distribution $T$ from equation (31) whereas the use of (35) and (38) in the equation (31) provides a term with factor $\left(1-\frac{R_{e} P_{r}}{v^{\prime}}\right)$ therefore it guides us to search for the function $B$ as solution of equation (49) of the type

$$
B(r, \psi)=\left(1-\frac{R_{e} P_{r}}{v^{\prime}}\right) R(r)
$$

Where $R(r)$ is an unknown function to be determined. Utilizing equation (50) in equation (49), we get

$$
\begin{aligned}
& \left(1-\frac{R_{e} P_{r}}{v^{\prime}}\right)\left[r Y R^{\prime \prime}+R^{\prime}\left(2 r Y^{\prime}+Y\right)+R\left(r Y^{\prime \prime}+Y^{\prime}\right)\right] \\
& +\frac{c R_{e} P_{r}}{v^{\prime}}\left[-(1+2 M Y) R^{\prime}-R\left(-\frac{c\left(1-M^{2}\right) Y}{r}+\frac{c M}{r}+2 M Y^{\prime}+Y M^{\prime}\right)\right] \\
& =R_{e}\left(\frac{1}{v^{\prime 2}}\right)\left[\frac{M^{\prime}}{r}+\frac{c\left(1+M^{2}\right)}{r^{2}}\right]+R_{e}\left(F_{1}\right)_{v}+R_{e} M\left(F_{2}\right)_{v}-R_{e}\left(r F_{2}\right)_{r}
\end{aligned}
$$

Here equation (51) is to provide the function $R(r)$, but it involves the components of unknown body force $F_{1}(r, \psi)$ and $F_{2}(r, \psi)$ therefore its solution depends upon the form of $F_{1}$ and $F_{2}$. We can select many possible forms of $F_{1}$ and $F_{2}$ leading to the solution of equation (49) for $R(r)$, however we find that not all arbitrarily selected forms lead to the solution of the momentum equations (29-30) for the function $L$ and the energy equation (31) for $T$. Our search for the appropriate form of $F_{1}$ and $F_{2}$ revealed that the solution of the momentum equations (29-30) and energy equation (31) is obtainable when the function $F_{2}(r, \psi)=F_{2}(r)$ is a solution of the following differential equation

$$
R_{e}\left(\operatorname{ar} F_{2}\right)_{r}=-\left[r\left(\begin{array}{ll}
Y & R
\end{array}\right)^{\prime}\right]^{\prime}
$$

Or

$$
R_{e} F_{2}=-\left(\begin{array}{ll}
Y & R
\end{array}\right)^{\prime}+\frac{h_{1}}{r}
$$

Where $h_{1}$ is constant.

On substituting equation (53) in equation (51) utilizing equation (41) and solving for $F_{1}$, we obtain

$\left.R_{e} F_{1}=\left(\frac{R_{e} P_{r}}{c K_{1}}\right)\left[\begin{array}{lll}r(Y & R\end{array}\right)^{\prime}\right]^{\prime} e^{-c v}$

$+\frac{R_{e} P_{r} e^{-c V}}{K_{1}}\left[(1+2 M Y) R^{\prime}+R\left(-\frac{c\left(1-M^{2}\right) Y}{r}+\frac{c M}{r}+2 M Y^{\prime}+Y M^{\prime}\right)\right]$

$+\left(\frac{R_{e} e^{-2 c v}}{2 c K_{1}^{2}}\right)\left[\frac{M^{\prime}}{r}+\frac{c\left(1+M^{2}\right)}{r^{2}}\right]^{\prime}+H(r)$

On substituting equations (53-54), in equations (29-30) and solving for the function $L$, we find

$$
\begin{aligned}
& R_{e} L=v h_{1}+\left(\frac{R_{e} e^{-2 c v}}{2 c K_{1}^{2}}\right)\left[\frac{M^{\prime}}{r}+\frac{c\left(1+M^{2}\right)}{r^{2}}\right]+\frac{\left[r(Y R)^{\prime}\right]}{c}\left(\frac{R_{e} P_{r} e^{-c v}}{K_{1}}\right) \\
& +(M Y+1) R\left(\frac{R_{e} P_{r} e^{-c v}}{K_{1}}\right)+
\end{aligned}
$$$$
\int\left(\frac{M h_{2}}{r}+2 M(Y R)^{\prime}+H(r)\right) d r+h_{2}
$$

Where $h_{2}$ is constant.

We can obtain viscosity from either of equations (32) or (33)

$$
\mu=-\frac{v^{\prime} r^{2}}{4(-1+c M)}\left(1-\frac{R_{e} P_{r}}{v^{\prime}}\right) R(r)
$$

The energy equation (31) on using (35), (38), (47), (50) and (56) becomes

$$
\begin{aligned}
& \left(r v^{\prime}\right) T_{r r}-2 M T_{v r} v^{\prime}+\frac{\left(1+M^{2}\right)}{r} T_{v v}\left(v^{\prime}\right)+\left(v^{\prime}-R_{e} P_{r}\right) T_{r}-M^{\prime} T_{v} v^{\prime} \\
& =-\frac{E_{c} P_{r}(-1+c M)}{r}\left(1+4 Y^{2}\right)\left(1-\frac{R_{e} P_{r}}{v^{\prime}}\right) R(r)
\end{aligned}
$$

Right-hand side of equation (57) suggests searching for solution of the type

$$
T(r, v)=\frac{K(r)}{v^{\prime}}
$$

Where $K(r)$ is unknown function to be determined. Utilizing equation (58) in equation (57) we find

$$
\begin{aligned}
& r K^{\prime \prime}+2 c M K^{\prime}+\frac{\left(1+M^{2}\right)}{r}\left(c^{2} K\right)+\left(1-\frac{R_{e} P_{r}}{v^{\prime}}\right) K^{\prime}+c K M^{\prime} \\
& =-\frac{E_{c} P_{r}(-1+c M)}{r}\left(1+4 Y^{2}\right)\left(1-\frac{R_{e} P_{r}}{v^{\prime}}\right) R(r)
\end{aligned}
$$


Comparing the coefficient of $\left(1-\frac{R_{e} P_{r}}{v^{\prime}}\right)$ on both side of equation (59), we get

$$
R(r)=-\frac{r K^{\prime}}{E_{c} P_{r}(-1+c M)\left(1+4 Y^{2}\right)}
$$

\section{And}

$$
r^{2} K^{\prime \prime}+2 c r M K^{\prime}+K\left[c^{2}\left(1+M^{2}\right)+c r M^{\prime}\right]=0
$$

The value of the unknown function $R(r)$ is to be determined from equation (60) but it involves another unknown function $K(r)$ satisfying equation (61). The coefficients of this equation depends on the function $M(r)=r f^{\prime}(r)$ and for one arbitrary choice of $f(r)$ the solution of equation (61) could be found using computer algebra system (CAS) software in terms of special functions. Finding $K(r)$ from equation (61) we find $R(r)$ from equation (60), $T$ from equation (58), viscosity $\mu$ from equation (56), pressure $p$ from (12) using (55) and velocity components from (8) for the components of body force $F_{1}$ and $F_{2}$ from (53-54). This indicates an infinite set of exact solutions however; we present here three examples of such exact solutions.

Example 1:

The equation (61) reduces to a Cauchy-Euler equation for

$$
M=m_{1}=\text { Const } .
$$

\section{Or}

$$
f(r)=m_{1} \ln r+m_{2}
$$

Where $m_{2}$ is constant of integration. Taking equation (62) in equation (61), we get

$$
r^{2} K^{\prime \prime}+2 m r K^{\prime}+\left(c^{2}+m^{2}\right) K=0
$$

Where

$$
m=c m_{1}=\text { Const } .
$$

Whose solution is

$$
K(r)=k_{3} r^{\lambda_{1}}+k_{4} r^{\lambda_{2}}
$$

Where

$$
\begin{aligned}
& \lambda_{1}=\frac{-(2 m-1)+\sqrt{1-4\left(m+c^{2}\right)}}{2} \\
& \lambda_{2}=\frac{-(2 m-1)-\sqrt{1-4\left(m+c^{2}\right)}}{2}
\end{aligned}
$$

Inserting equation (66-68) in equation (58), we get

$$
T(r, v)=\frac{\left(k_{3} r^{\lambda_{4}}+k_{4} r^{\lambda_{2}}\right)}{k_{1} e^{c v}}
$$

Thus for $K(r)$ from equation (66) we find $R(r)$ from equation (60), pressure $p$ using (55), viscosity $\mu$ from equation (56) and $T$ from equation (69), viscosity $\mu$ from equation (56), pressure $p$ from (12) using (55) and velocity components from (8) for the components of body force $F_{1}$ and $F_{2}$ from (53-54).

\section{Example 2:}

In order to reduce the order of equation (61) we set

$c\left(1+M^{2}\right)+r M^{\prime}=0$

Or

$M(r)=\operatorname{Tan}\left(m_{3}-c \ln r\right)$

Substituting (71) in equation (46) and solving the resulting differential equation, we find

$f(r)=\frac{1}{c} \ln \cos \left(m_{3}-c \ln r\right)+m_{4}$

Taking equation (72) in equation (61), we get

$r^{2} K^{\prime \prime}+2 c r \operatorname{Tan}\left(m_{3}-c \ln r\right) K^{\prime}=0$

Whose solution is

$K(r)=k_{5} \int \sec ^{2}\left(m_{3}-c \ln r\right) d r+k_{6}$

Inserting equation (74) in equation (58), we get

$T(r, v)=\frac{k_{5} \int s c e^{2}\left(m_{3}-c \ln r\right) d r+k_{6}}{k_{1} e^{c v}}$

Thus finding $K(r)$ from equation (74) we find $T$ from equation (75), $R(r)$ from equation (60), viscosity $\mu$ from equation (56), pressure $p$ from (12) using (55) for the components of body force $F_{1}$ and $F_{2}$ from (53-54).

Example 3:

Here we let solution of equation (61) is

$K(r)=U(r) V(r)$

Taking (76) in equation (61) and removing the first derivative term [18], we find

$U(r)=e^{-c f(r)}$

And

$r^{2} V^{\prime \prime}+V\left(c^{2}+c r f^{\prime}\right)=0$

A solution of (78) is

$V(r)=k_{7} \operatorname{Cos} r+k_{8} \operatorname{Sin} r$

When

$f(r)=\frac{r^{2}}{2 c}-c \ln r$

Therefore inserting (77) and (79) in (76), we get

$K(r)=r^{c^{2}} e^{-\frac{r^{2}}{2}}\left(k_{7} \operatorname{Cos} r+k_{8} \operatorname{Sin} r\right)$

And inserting equation (81) in equation (58), we find

$T(r, v)=\frac{r^{c^{2}} e^{\frac{r^{2}}{2}}\left(k_{7} \operatorname{Cos} r+k_{8} \operatorname{Sin} r\right)}{k_{1} e^{c v}}$ 
Where $k_{i}, i \in\{1,2,3,4,5,6,7,8\}$ and $m_{i}, i \in\{1,2,3,4\}$ are constants. Thus finding $K(r)$ from equation (81) we find $T$ from equation (82), $R(r)$ from equation (60), viscosity $\mu$ from equation (56), pressure $p$ from (12) using (55) for the components of body force $F_{1}$ and $F_{2}$ from (53-54). The streamline patterns can be drawn to observe the effect of various parameters for $c>0$ and $c<0$.

For the case $Y(r)=0$, the equation (48) implies

$r M^{\prime}-2 M-c\left(1-M^{2}\right)=0$

Since here, either $c>0$ or $c<0$ therefore we provide solution of equation (83) for $c=+1$ and $c=-1$ as an example and likewise one can find for $c>0$ or $c<0$. When $c=+1$ and $c=-1$, the equation (83) on utilizing equation (46) provides

$$
f(r)=c_{2}+\ln r+\ln \left[\cosh \left(\sqrt{2}\left(c_{1}-\ln r\right)\right)\right]
$$

And

$$
f(r)=c_{2}-\ln r-\ln \left[\cosh \left(\sqrt{2}\left(c_{1}+\ln r\right)\right)\right]
$$

Respectively. Inserting equation (83) in equation (44), we find

$$
A=0
$$

Using (86) in (40), we get

$$
-\left\{B_{r}-\frac{f^{\prime} B_{\psi}}{v^{\prime}}\right\}_{\psi}=R_{e} w_{r}+R_{e}\left(F_{1}+F_{2} r\left(f^{\prime}\right)\right)_{\psi}-R_{e}\left(r v^{\prime} F_{2}\right)_{r}
$$

Here equation (87) is to provide the function $B$, but it involves the components of unknown body force $F_{1}(r, \psi), F_{2}(r, \psi)$ and vorticity function $w$ therefore its solution will depend upon the form of these functions. We can select many possible forms of these functions leading to the solution of equation (87) for $B$. However, we find that arbitrarily selected forms of these functions do not lead to the solution of the momentum equations (29-30) for the function $L$ and the energy equation (31) for $T$. One successful search for the form of the functions $F_{2}(r, \psi)$ is to take $F_{2}(r, \psi)=F_{2}(r)$ satisfying

$$
R_{e}\left(r v^{\prime} F_{2}\right)_{r}=R_{e} w_{r}
$$

Or

$$
R_{e}\left(r v^{\prime} F_{2}\right)=R_{e} w+G(\psi)
$$

Where $G(\psi)$ is function of integration and vorticity function $w$ is arbitrary. Utilizing (89) in (87), we get

$$
R_{e}\left(F_{1}+F_{2} r\left(f^{\prime}\right)\right)_{\psi}=-\left\{B_{r}-\frac{f^{\prime} B_{\psi}}{v^{\prime}}\right\}_{\psi}
$$

Or

$$
R_{e} F_{1}=-R_{e} F_{2} r\left(f^{\prime}\right)-\left\{B_{r}-\frac{f^{\prime} B_{\psi}}{v^{\prime}}\right\}+H_{1}(r)
$$

Where $H_{1}(r)$ is a function of integration. On substituting equations (89) and (91), in equations (29-30) and solving for the function $L$, we have

$$
R_{e} L=-B-\int G(\psi) d \psi+\int H_{1}(r) d r+h_{3}
$$

Where $h_{3}$ is constant of integration.

Now the energy equation (30) on using equation (86), becomes

$$
\begin{aligned}
& r T_{r r}-2 M T_{v r}+\frac{\left(1+M^{2}\right)}{r} T_{v v} \\
& +\left(1-\frac{R_{e} P_{r}}{v^{\prime}}\right) T_{r}-M^{\prime} T_{v}=-\frac{r E_{c} P_{r}}{4 \mu}\left(B^{2}\right)
\end{aligned}
$$

Substituting equation (45) in equation (93), we find a relation for viscosity in terms of the derivative of the temperature function $T$

$$
\mu=\left(\frac{-r^{3}\left(v^{\prime}\right)^{2}}{4 E_{c} P_{r}(c M-1)^{2}}\right)\left[\begin{array}{l}
r T_{r r}-2 M T_{v r} \\
+\frac{\left(1+M^{2}\right)}{r} T_{v v} \\
+\left(1-\frac{R_{e} P_{r}}{v^{\prime}}\right) T_{r}-M^{\prime} T_{v}
\end{array}\right]
$$

Where the function $M(r)=r f^{\prime}(r)$ is according to the equations (84) and (85).

The streamline patterns can be drawn to observe the effect of various parameters for $c>0$ and $c<0$.

\section{Results and discussion}

We have found some new exact solutions of the system of partial differential equations governing the steady, plane motion of incompressible fluid of variable viscosity with body force term to the right-hand side of Navier-Stokes equations of a class. The class consists of the stream function $\psi$ characterized by $\theta=f(r)+\frac{1}{c} \ln \left[\frac{-1}{c\left(k_{1} \psi+k_{2}\right)}\right]$ in polar coordinates $r$ and $\theta$ where a continuously differentiable function is $f$ and the constant $c>0$ or $c<0$. For both values of the constant $c$, we found solutions for given one component of the body force for arbitrary $f(r)$ which indicates an infinite set of exact solutions to the problem concerned however, we mentioned three example exact solutions. When the case fixes the function $f(r)$ we find viscosity as derivative of function of temperature for $c>0$ or $c<0$ and mention such relation for $c=+1$ and $c=-1$ as an example. In both the cases when $f(r)$ is arbitrary and when it is not, we indicated an infinite set of streamlines, the velocity components, viscosity function, generalized energy function and temperature distribution. For $c>0$ and $c<0$ the streamline patterns can be drawn using CAS to observe the effect of various parameters.

\section{References}

[1] Mushtaq A., On Some Thermally Conducting Fluids: Ph. D Thesis, Department of Mathematics, University of Karachi, Pakistan, 2016.

[2] Mushtaq A.; Naeem R.K.; S. Anwer Ali; A class of new exact solutions of Navier-Stokes equations with body force for viscous incompressible fluid, International Journal of Applied Mathematical $\begin{array}{llll}\text { Research, } & 2018, & 7 & \text { (1), }\end{array}$ http:/www.sciencepubco.com/index.php/IJAMR. https://doi.org/10.14419/ijamr.v7i1.8836.

[3] Naeem, R. K.; Mushtaq A.; A class of exact solutions to the fundamental equations for plane steady incompressible and variable viscosity fluid in the absence of body force: International Journal of Basic and Applied Sciences, 2015, 4 (4), 429-465 http:/www.sciencepubco.com/index.php/IJBAS https://doi.org/10.14419/ijbas.v4i4.5064.

[4] Wang, C. Y.; on a class of exact solutions of the Navier-Stocks equations: Journal of Applied Mechanics, 33 (1966) 696-698. https://doi.org/10.1115/1.3625151.

[5] Kapitanskiy, L.V.; Group analysis of the Navier-Stokes equations in the presence of rotational symmetry and some new exact solutions: Zapiski nauchnogo sem, LOMI, 84 (1) (1979) 89-107. 
[6] Dorrepaal, J. M.; an exact solution of the Navier-Stokes equations which describes non-orthogonal stagnation -point flow in two dimensions: Journal of Fluid Mechanics, 163(1) (1986) 141-147. https://doi.org/10.1017/S0022112086002240.

[7] Chandna, O. P., Oku-Ukpong E. O.; Flows for chosen vorticity functions-Exact solutions of the Navier-Stokes Equations: International Journal of Applied Mathematics and Mathematical Sciences, $17 \quad$ (1) 1994) 155-164 https://doi.org/10.1155/S0161171294000219.

[8] Naeem, R. K.; Exact solutions of flow equations of an incompressible fluid of variable viscosity via one - parameter group: The Arabian Journal for Science and Engineering, 1994, 19 (1), 111-114.

[9] Naeem, R. K.; Anwer Ali, S.; Exact solutions of the equations of motion of an incompressible fluid of variable viscosity: Karachi University Journal of Science, 1996, 24 (1), 35-40.

[10] Naeem, R. K.; Srfaraz, A. N.; Study of steady plane flows of an incompressible fluid of variable viscosity using Martin's System Journal of Applied Mechanics and Engineering, 1996, 1 (1), $397-$ 433.

[11] Naeem, R. K.; Anwer Ali, S.; A class of exact solutions to equations governing the steady plane flows of an incompressible fluid of variable viscosity via von-Mises variables: International Journal of Applied Mechanics and Engineering, 2001, 6 (1), 395-436.

[12] Naeem, R. K.; Steady plane flows of an incompressible fluid of variable viscosity via Hodograph transformation method: Karachi University Journal of Sciences, 2003, 3 (1), 73-89.

[13] Naeem, R. K.; on plane flows of an incompressible fluid of variable viscosity: Quarterly Science Vision, 2007, 12 (1), 125-131.

[14] Naeem, R. K.; Sobia, Y.; Exact solutions of the Navier-Stokes equations for incompressible fluid of variable viscosity for prescribed vorticity distributions: International Journal of Applied Mathematics and Mechanics, 2010, 6 (5), 18-38.

[15] Giga, Y.; Inui, K.; Mahalov; Matasui S.; Uniform local solvability for the Navier-Stokes equations with the Coriolis force: Method and application of Analysis, 2005, 12, 381-384.

[16] Gerbeau, J. -F.; Le Bris, C., A basic Remark on Some NavierStokes Equations with Body Forces: Applied Mathematics Letters 2000, 13 (1), 107-112. https://doi.org/10.1016/S08939659(99)00194-9.

[17] Martin, M. H.; The flow of a viscous fluid I: Archive for Rational Mechanics and Analysis, 1971, 41 (4), 266-286. https://doi.org/10.1007/BF00250530.

[18] Daniel Zwillinger; Handbook of differential equations; Academic Press, Inc. (1989)]. 